\title{
Co-Designing Contextual Tutorials for Older Adults on Searching Health Information on the Internet
}

\author{
Bo Xie ${ }^{1}$ \\ Tom Yeh ${ }^{2}$ \\ Greg Walsh ${ }^{1}$ \\ Ivan Watkins ${ }^{1}$ \\ ${ }^{1}$ College of Information Studies, University of Maryland \\ ${ }^{2}$ Department of Computer Science, University of Maryland \\ College Park, MD 20742
}

Man Huang ${ }^{1}$

\{boxie@umd.edu, tomyeh@umd.edu, gwalsh@umd.edu, iwatkins@umd.edu, manhuang@umd.edu\}

\begin{abstract}
Evidence in the literature suggests an integrated e-tutorial is more effective than a paper- or video-based tutorial among younger people. Yet, relatively little is known about the effectiveness of an integrated e-tutorial on the older population. This study explored the applicability of an integrated e-tutorial to an older population, focusing on the content area of e-health literacy. A specific integrated etutorial, the Online Tutorial Overlay Presenter (OnTOP), was used to add an overlay to the NIHSeniorHealth.gov Website. Features of the overlay were examined thoroughly in seven 2-hour-long participatory design sessions with ten older adults. Several participatory design techniques were used to elicit participants' preferences for design features of the OnTOP tutorial. These techniques included drawing on the board, voice recording and integration, and peer instruction. Three major themes emerged: 1) using contextual cues to facilitate learning; 2) tailoring to accommodate the learner's literacy level; and 3) enhancing existing interfaces with multimedia cues. These findings improved the design of OnTOP. They also contribute to the multimedia learning literature, generating empirical evidence about the effects of multimedia learning among the understudied older adult population.
\end{abstract}

\section{Keywords}

Integrated e-tutorial, e-health literacy, participatory design, health information seeking.

\section{INTRODUCTION}

Locating useful information online is an important skill for older adults, despite their low Internet literacy rates (Xie, 2008). This study investigates effective tutorial design for teaching older adults, building on the multimedia learning,

ASIST 2011, October 9-13, 2011, New Orleans, LA, USA

instructional design, and participatory design literatures.
Traditionally, tutorials designed for older adults use print or video media (Echt et al., 1998; Hawthorn, 2007). These media require alternating between two contexts: the presentation context, where the information is presented, and the application context, where knowledge is applied in computer operation. Working with two contexts poses several challenges in that older adults must: 1) constantly alternate between the contexts; 2) reconcile content between the contexts; and 3) rely on short-term memory to transfer instructions from the presentation context to the application context. Merging these contexts into one application interface provides a technical solution to these challenges. Instead of presenting the instructions in separate contexts, this solution provides instructions in the target application. For instance, this solution highlights links and provides explanatory text on the screen itself. The literature indicates this approach benefits user populations like general computer users (Kang \& Plaisant, 2005), but no known study tested older adult users.

Older adults are typically not involved in technology design (Paquette \& Xie, 2010). To fully address older adults' technological needs, older adults must be involved in the design process (Xie, et al., 2010). This study involved older adults in the design process through participatory design.

\section{METHOD}

Research site

This study took place at a public library in Maryland between November 2010 and March 2011.

\section{Design Partners}

Seven older adults participated in this study. Table 1 below summarizes their gender, age, and computer use frequency.

\begin{tabular}{|l|l|l|}
\hline $\begin{array}{l}\text { Code } \\
\text { name }\end{array}$ & Age & Computer Use Frequency \\
\hline Mr. RH & 88 & Every 2-3 days \\
\hline Ms. MC & 62 & Every day \\
\hline Ms. RG & 72 & Every 2-3 days \\
\hline Ms. JML & 80 & Every day \\
\hline Mr. NJ & 61 & Every 2-3 days \\
\hline Ms. JS & 77 & Every day \\
\hline Ms. SG & 61 & Every 2-3 days \\
\hline
\end{tabular}

Table 1. Participant background information. 


\section{Design Process}

This study consisted of three phases: introduction, simulation design, and prototyping. We ran seven, two-hour participatory design sessions over three phases (Table 2).

\begin{tabular}{|l|l|l|}
\hline Phase & Session & Activity \\
\hline $\begin{array}{l}\text { Phase 1: } \\
\text { Introduction }\end{array}$ & 1 & $\begin{array}{l}\text { OnTOP presented, solicited } \\
\text { design suggestions }\end{array}$ \\
\cline { 2 - 3 } & 2 & Changes to OnTOP presented \\
\hline $\begin{array}{l}\text { Phase 2: } \\
\text { Simulation }\end{array}$ & 3 & $\begin{array}{l}\text { Recorded participants reading } \\
\text { verbal instructions out loud }\end{array}$ \\
\cline { 2 - 3 } & 4 & $\begin{array}{l}\text { Presented OnTop with } \\
\text { participants' narration }\end{array}$ \\
\cline { 2 - 3 } & 5 & $\begin{array}{l}\text { Physical context: Participants } \\
\text { provide instructions to each } \\
\text { other while seated at the } \\
\text { computer }\end{array}$ \\
\hline $\begin{array}{l}\text { Phase 3: } \\
\text { Prototyping }\end{array}$ & 6 & $\begin{array}{l}\text { Lo-fidelity prototyping using } \\
\text { annotated storyboards }\end{array}$ \\
\cline { 2 - 3 } & 7 & $\begin{array}{l}\text { Target identification using } \\
\text { different types of references }\end{array}$ \\
\hline
\end{tabular}

Table 2. Activities used in the co-design phases and sessions of the present study.

\section{Design Phase 1: Introduction}

Phase 1 familiarized participants with participatory design. In the first session, we presented a prototype of our contextual tutorial tool, Online Tutorial Overlay Presenter (OnTOP), and solicited participants' design ideas. The tutorial's content is based on a paper-based tutorial that teaches older adults to use NIHSeniorHealth.gov.

OnTOP is a contextualized version of the paper-based tutorial. It displays the same text instructions and visual annotations (e.g., highlight) as the paper-based tutorial, but integrates them into the website's interface. For example, the paper-based tutorial asks users to click on Diabetes. With OnTOP, a highlight appears on the actual "Diabetes" link, along with a text box instructing users to "click on Diabetes." Users can click the link. Users took turns practicing with the OnTop prototype.

After this session, we improved the prototype based on participants' suggestions, and presented the revised prototype in the next session to elicit further feedback.

\section{Design Phase 2: Simulation Design}

Phase 2 focused on gaining insight into the desired features of contextual help through simulation design. We asked participants to speak contextual help aloud, simulating what they wanted OnTOP to include.

In the first session, participants practiced giving verbal instructions, reading paper-based tutorial instructions aloud while we recorded their speech. We instructed the participants to pretend to instruct another person while reading the script. In the second session, we asked participants to modify the instructions for clarity. The participants took turns recording their voices using the improved script. Afterwards we integrated the recorded voices into OnTOP. In the last session, participants provided contextual help without a script. Participants paired up, with one participant acting as instructor and one as student. Participants switched roles, repeating the exercise with a different topic.

\section{Design Phase 3: Prototyping}

In this phase, participants designed paper prototypes using pens and highlighters. In the first session, we examined how a contextual tutorial could integrate contextualized annotations (e.g., a text bubble) into an interface. We provided users with a series of screenshots of NIHSeniorhealth.gov on separate pieces of paper. We provided participants with pens and highlighters, asking them to add annotations to the screenshots, as if they were adding annotations contextually to the Web interface. Participants also arranged screenshots in the order they found useful. Participants were told the goal was developing instructions for locating information about diabetes on the NIHSeniorhealth.gov website for a friend. This activity helped researchers learn about how participants would develop a contextual tutorial, observing the symbols, language, and highlights participants used.

In the final session we examined how a contextual tutorial could provide different references to help users identify interface targets on the computer screen. We examined four reference types: text, image, image with surrounding text, and image with embedded text (Table 3 ).

\begin{tabular}{|c|c|}
\hline Exemplar reference & $\begin{array}{l}\text { Type of } \\
\text { reference }\end{array}$ \\
\hline \multirow[t]{2}{*}{$\begin{array}{l}\text { Free interactive tools to check your health, get } \\
\text { personalized advice, and keep track of your progress. }\end{array}$} & Text \\
\hline & Image \\
\hline $\begin{array}{l}\text { Diabetes } \\
\text { Do you have diabetes or } \\
\text { pre-diabetes? }\end{array}$ & $\begin{array}{l}\text { Image with } \\
\text { surrounding } \\
\text { text }\end{array}$ \\
\hline & $\begin{array}{l}\text { Image with } \\
\text { embedded } \\
\text { text }\end{array}$ \\
\hline
\end{tabular}

Table 3. List of tasks performed by participants during the testing of HealthFinder.gov in Session 7.

First, we wanted participants to experience different reference types. We designed target identification tasks using Healthfinder.gov, which participants had never used. Each task consisted of a color screenshot and a reference to a target. For example, one task asked participants to "Locate INTERACTIVE TOOLS" on a screenshot. Healthfinder.gov's unfamiliarity forced participants to rely on the references to locate objects. This technique helped 
us assess the references' effectiveness without the potentially confounding factor of prior knowledge.

\section{Data Analysis}

Inductive thematic analysis guided the data analysis (Braun \& Clarke, 2006). Inductive thematic analysis aims to identify salient themes, which was appropriate for this study's exploratory nature.

\section{KEY FINDINGS}

Three major themes emerged: 1) contextual cues facilitating learning; 2) the learners' diverse literacy Levels; and 3) enhancing existing interfaces.

\section{Contextual Cues Facilitating Learning}

Gestural cues to help focus attention

Sharing a physical context helped participants focus each other's attention. This occurred in the simulated contextual help setting, when participants accompanied verbal explanations with gestural cues. For example, Mr. $\mathrm{RH}$ stated, "we get 'bones and joints' by clicking on the first category," while pointing at the "bones and joint category". Mr. RH's use of gestural cues relieved the need for purely verbal explanation.

Recognizing gestural cues' benefit, we solicited ideas for achieving this effect on the computer. Mr. RH suggested using an apple to focus users' attention on the screen, presumably because of the symbols' familiarity and color.

Providing immediate feedback using confirmation cues Confirmation cues, provided in context, can help participants recognize when they complete a task. For example, a confirmation cue could indicate a participant clicked the correct link. Participants provided useful design ideas for building confirmation cues on the computer.

\section{The Learners' Diverse Literacy Levels}

Providing instructions with appropriate level of details Participants suggested the detail level of the tutorial should correspond to the users' knowledge level. Mr. NJ stated, "for [the] person that doesn't know how to use the computer, you need to show [them] every function." More advanced users would not require this detailed instruction.

\section{Lack of Sufficient Computer Knowledge}

Computer tutorials use commonly accepted terms to reference interface elements, which is problematic for users with limited knowledge. For example, some participants could not find text-based links or image-based symbols. They did not understand the text-based links like "back to top" or the image-based symbols like the search button. Conventionally, the "Back to Top" symbol means "click here to scroll back to the top of the page" while the search button typically commences the search process. Participants did not understand these symbols. Similarly, users may have difficulty with unfamiliar terminology. For instance, Ms. RG referred to the cursor as "the little hand."

Participants recognized the importance of conventions when developing their version of the tutorial. When deciding how to number different steps, participants argued about using Roman numerals. Participants chose Arabic numeral because Roman numerals might be unfamiliar.

\section{Enhancing Existing Interfaces}

Participants identified many ways to enhance the existing interface of websites or tutorials.

\section{Adding custom images and animations}

Participants wanted to enhance text with illustrative images. For example, Ms. SG stated, "we [put] a picture of a syringe... next to the diabetes link." Ms. SG felt that this illustration clarified the link's meaning. Similarly, participants suggested replacing text with illustrations. For example, Ms. SG drew people talking for the "speech button" Mr. RH similarly felt illustrations improved the tutorial's clarity. Illustrations may reduce reliance on textual explanations and reinforce visually conveyed information.

\section{Adding extra explanations}

Participants suggested adding information for clarification. For example, one participant suggested editing the instruction "Open a Web browser," to "Open a web browser, for example, try Internet Explorer, " arguing a user may not know "Web browser" but would know Internet Explorer. Providing the additional information increases clarity while explaining what a Web browser is.

\section{Adding reading aids}

Participants found small text difficult to read. They suggested a text enlargement feature that would aid older users. While most browsers support this feature, users, may not be aware of it. One solution is automatically detecting when the font is too small and instruct the users to enlarge the font.

\section{DISCUSSION}

This study contributes to the multimedia learning literature. Participants favored auditory instructional presentation, supporting the modality principle (Low \& Sweller, 2004).

Interestingly, their positive attitude towards the auditory presentation mode persists even when the narration is identical to the written instructions. This seems contradictory to the redundancy principle that predicts negative impact when identical information is presented in multiple media forms (Mayer, 2005b). A closer look, however, suggests a new possibility: that identical information presented visually and verbally may not be "redundant". This would be in line with the findings of a randomized controlled experiment study, conducted at the same library site, which revealed no statistically significant difference in the learning outcomes of older adults in groups receiving visual only instructions and those receiving visual plus auditory instructions (Xie, in press).

However, the site where the experiments took place might have affected the outcomes (Xie, in press). The library site lacks a computer lab, so sessions were held in a public area where other patrons walked and sometimes talked. These 
distractions might interfere with older adults' learning, particularly for the visual only condition. In comparison, for the visual plus auditory condition, because the auditory information was presented directly via headphones, hearing the narration may have supported participants' focus. In this sense, the information presented via the auditory channel might not have been "redundant" to that presented visually (Xie, in press). Because this study occurred at the same site, it might have had a similar effect.

The findings of this study support the signaling principle (Mayer, 2005). Participants relied on gestural and confirmation cues to provide instructions, and recommended ways to incorporate these cues into the OnTOP tutorial. These findings are in line with prior signaling principle research (Mautone \& Mayer, 2001).

The multimedia learning literature focuses primarily on younger learners in formal educational settings (Mayer, 2005a). Our findings suggest multimedia learning theories may be generalized to the older population in informal settings. Our findings also provide empirical evidence for the proposed design approaches under the cognitive aging principle (Paas, et al., 2005), the only known multimedia learning principle targeting older learners. In particular, the OnTOP tutorial ensures learner controlled pacing, which can accommodate individualized differences.

This study had limitations: (1) small sample size and (2) sessions were conducted in a naturalistic setting (a library) which might have introduced some confounding factors (e.g., environmental noise).

\section{CONCLUSION}

Using various participatory design techniques, we explored older adults' preferences for design features of an integrated e-tutorial, OnTOP. The findings suggest contextual cues, including gestural and auditory cues, may facilitate learning. Due to the diversity of learner literacy, it is important to provide tutorials tailored to learners' literacy. Further, tutorial interfaces may be enhanced with multimedia cues.

These findings improve OnTOP's design features and contribute to the multimedia learning literature by generating empirical evidence about the effects of multimedia learning among older adults, a previously understudied population in the literature, and by raising interesting questions for further examination.

\section{REFERENCES}

Braun, V., \& Clarke, V. (2006). Using thematic analysis in psychology. Qualitative Research in Psychology, 3, 77101.

Echt, K. V., Morrell, R. W., \& Park, D. C. (1998). Effects of age and training formats on basic computer skill acquisition in older adults. Educational Gerontology, 24(1), 3-25.
Hawthorn, D. (2007). Interface design and engagement with older people. Behaviour \& Information Technology, 26(4), 333-341.

Kang, H. and Plaisant, C. New approaches to help users get started with visual interfaces: multi-layered interfaces and integrated initial guidance. In Proceedings of the 2003 Annual National Conference on Digital Government Research, (2003).

Low, R. \& Sweller, J. (2005). The Modality Principle in Multimedia Learning. In R. Mayer (Ed.), The Cambridge Handbook of Multimedia Learning (pp. 183-200). New York: Cambridge University Press.

Mautone, P. D., \& Mayer, R. E. (2001). Signaling as a cognitive guide in multimedia learning. Journal of Educational Psychology, 93, 377-389.

Mayer, R. E. (2005a). The Cambridge Handbook of Multimedia Learning (ed.). Cambridge University Press: New York.

Mayer, R. (2005b) Principles for Reducing Extraneous Processing in Multimedia Learning: Coherence, Signaling, Redundancy, Spatial Contiguity, and Temporal Contiguity Principles. In R. Mayer (Ed.), The Cambridge Handbook of Multimedia Learning (pp. 183-200). New York: Cambridge University Press.

Moraveji, N., Li, J., Ding, J., O’Kelley, P., \& Woolf, S. (2007). Comicboarding: using comics as proxies for participatory design with children. Proceedings of the SIGCHI conference on Human factors in computing systems, CHI '07 (p. 1371-1374). New York, NY, USA: ACM. doi:10.1145/1240624.1240832

Paas, F., Van Gerven, P., \& Tabbers, H. (2005). The Cognitive Aging Principle in Multimedia Learning. In R. Mayer (Ed.), The Cambridge Handbook of Multimedia Learning (pp. 339-351). New York: Cambridge University Press.

Paquette, S. \& Xie, B. (2010). The Relevance of Elderly Technology Users in Healthcare Knowledge Creation and Innovation: A Case Study. In Proceedings of the $43^{\text {rd }}$ Hawaii International Conference on Systems Sciences. January 5-8, 2010, Hawaii.

Xie, B. (2008). Older adults, health information, and the Internet. ACM Interactions, 15(4), 44-46.

Xie, B. (in press). Experimenting on the impact of learning methods and information presentation channels on older adults' e-health literacy. Journal of the American Society for Information Science and Technology (JASIST).

Xie, B., Druin, A., Fails, J., Massey, S., Golub, E., Franckel, S., \& Schneider, K. (2010). Connecting generations: developing co-design methods for older adults and children. Behaviour \& Information Technology. 